\title{
Clinical Study Remineralization Effect of Mineralization Varnish
}

\author{
Dr Dobrinka Damyanova ${ }^{1}$, Dr Sirma Angelova ${ }^{1}$, \\ Dr Teodora Targova-Dimitrova Phd ${ }^{2}$ \\ ${ }^{1}$ Assistant Professor, PhD student, Medical University-Varna, Bulgaria, Faculty of Dental Medicine, \\ Department of Pediatric Dental Medicine \\ ${ }^{2}$ Assistant Professor, Medical University-Varna, Bulgaria, Faculty Of Dental Medicine, Department of \\ Pariodotology And Dental Implantology
}

\begin{abstract}
:
Introduction: Several studies point out that dental products, such as fluoride varnishes, are able to promote remineralization and to control the development of the carious process.

Purpose: To evaluate the effectiveness of fluoropolymers varnishes used for prophylaxis and non-invasive treatment of initial caries $\mathrm{d} 1, \mathrm{~d} 2$ in children 4 to 6 years.

Material and Methods: Non-invasive treatment of lesions d1a, d1b and d2. After processing results and highlights of the actual study was conducted by the data processing package for mathematical and statistical analysis SPSS v 20.0.

Results: In a 4-year-olds observed at (2.7 times higher), and the average lesion before treatment was 11,35 \pm $2,41(\mathrm{t}=12,43, \mathrm{p}<0.001)$.

At 5 and 6 year olds difference before and after treatment was 2.6 times the average value of the lesions prior to treatment with 5 year is $11,25 \pm 2,47$, and at 6-year was $11,15 \pm 2,23$ (respectively $t=12,01, \mathrm{p}<0,001$ for 5 years and $t=12,93, p<0,001$ for 6 years).

Conclusion: Clinpro TM White Varnish with TCP (Tri-Calcium phosphate) (3M) is effective in reducing demineralization in subsurface layer.
\end{abstract}

Keywords: remineralization, caries lesion, mineralization varnish

\section{Introduction}

Several studies point out that dental products, such as fluoride varnishes, are able to promote remineralization and to control the development of the carious process[1], to reduce demineralization adjacent to orthodontic brackets[2], and to reduce caries.

Objectives: To evaluate the effectiveness of fluoropolymers varnishes used for prophylaxis and noninvasive treatment of initial caries $\mathrm{d} 1, \mathrm{~d} 2$ in children 4 to 6 years.

Tasks: To investigate the clinical curative effect and remineralization of mineralization varnish - Clinpro TM White Varnish with TCP (Tri-Calcium phosphate) (3M) for a period of 12 months with DIAGNOdent Pen - in vivo.

\section{Material and Methods}

The study reporting the therapeutic effect of Tri - Calcium Phosphat, Clinpro White Varnish fluorine varnish for a period of 12 months with DIAGNOdent Pen - in vivo include 100 children in total, 50 girls and 50 boys - (to age 4 years 30 children aged 5 years to 10 children aged six years and 60 children). Carry out controlled clinical studies comparing the use of a fluorine varnish - Clinpro TM White varnish with TCP, which is applied during the first month of each week, and then at an interval of 3 months over a period of one year. In the control group included 100 children, 50 girls and 50 boys of the same age who have applied non-operative treatment or fluorine medication. The duration of the trial was 12 months. The fluorine varnish is applied to the vestibular caries lesions- d1a, d1b, and d2, diagnosed with DIAGNOdent Pen. Treated with varnish 400 teeth with lesions $\mathrm{d} 1 \mathrm{a}, \mathrm{d} 1 \mathrm{~b}$ and $\mathrm{d} 2-$ common all age groups. Conduct and organization of study. Survey period 2014 - 2015. The study is realized in the Faculty of Dental Medicine - Varna. The study has been authorized by the Commission on the Ethics of Scientific Research at MU Varna and was taken in advance declared informed consent from each parent, or of any child patient. After processing results and highlights of the actual study was conducted by the data processing package for mathematical and statistical analysis SPSS v 20.0. in the period March - May 2015.

Methods and criteria for the performance of the treatment. Different is the effect of treatment for lesions d1a - complete cure, but d1b and d2 lesions - detention and stop the development and deepening them. 
Because of the easy methods for children, the composition of the mineralized varnish and successful in vitro and clinical results we selected Application application for noninvasive treatment of lesions with d1 varnish Clinpro TM White Varnish with TCP (Tri-Calcium phosphate) (3M). To evaluate the effectiveness of treatment methods such as clinical examination of colored enamel porosity, lesion size, presence of demarcation with healthy enamel, methods of visualization solutions, radiographic methods and clinical study with DIAGNOdent Pen. Porosity examine probe WHO-621. We apply modern methods of diagnosis and treatment of primary dental lesions.

Non-invasive treatment for lesions d1a. Values obtained with DIAGNOdent Pen 4-6 Conduct monitoring and evaluation of lesions and oral hygiene. Local fluoridation Clinpro TM White Varnish with TCP, application once a 1-st week. When normalized lesions and type of enamel surface, control the polish of three months during the first year. Mastering process - normal control six months. In the absence of a result produce a new evaluation of risk factors and choice of measures for their modeling.

Non-invasive treatment of lesions $\mathrm{d} 1 \mathrm{~b}$ and $\mathrm{d} 2$. Values obtained with DIAGNOdent Pen 7-15, 15-20. Treatment for lying on vestibular surfaces smooth in the cervix. Local fluoridation with Clinpro TM White Varnish with TCP is application once a 1-st week for a month. When normalized lesions and type of enamel surface, control and polish of three months during the first year. Individual remineralization with Tooth Mousse is held every evening after personal hygiene and without washing. Held three months. Control for two weeks to assess the lesion, and the activity of the process.

Professional treatment continues to onset of visual signs of stationing of the lesion. It aims to achieve a reduction in the values of DIAGNOdent Pen to $=2$. In the absence of regression or stationing, a new assessment of risk factors and a redefinition of the treatment plan.

\section{Clinical application and application of mineralized varnish. Made described below steps.}

1)Zone (s) to be treated and helps prevent, polished brush for corner angle in the clinical setting. Used brush Applicator good mix of white lacquer Clinpro, since all the components of the varnish with sodium fluoride can be separated during storage.

2) Applicated in white varnish Clinpro evenly over the treated area.

3) The period of treatment with a white lacquer Clinpro be at least 4 hours, preferably overnight.

Children are reviewed with a history and detailed registration of clinical status and plaque index OHI-S = PLI-S Green-Vermillion (JC Green, JRVermillion, 1964) (modified) and evaluation of gingival bleedingPapilla Bleeding Index (PBI) - Ainamo \& Bay (Simplified). After detailed in outpatient registration cards numbers in the clinical setting with polished precision brush each tooth surfaces smooth, then dried. Investigates any smooth surface-vestibular and visual oral, clinical methods, DIAGNOdent Pen, radiographs and register values obtained for each and every tooth surface the every child - patient.

\section{Results}

The results for detection with lesions d1 DIAGNOdent pen by the method of laser fluorescence of the control group at the beginning and end of each study. The results of the comparative analysis shows that the significant difference in the average value of the lesion before and after treatment of 1,3,6,9 and 12 months. DIAGNOdent Pen with children of the corresponding ages. In a 4-year-olds observed the average lesion before treatment was 4,90 $\pm 1,37(\mathrm{t}=5,56, \mathrm{p}<0,001)$. At 5 and 6 year olds difference before and after treatment is less than 2 times the average value of the lesions prior to treatment with 5 year was $5,10 \pm 1,41$, and at 6-year was 5,30 $\pm 1,34$ (respectively $t=5,94, \mathrm{p}<0,001$ for 5 years and $t=6,56, p<0,001$ for 6 years) Fig. 1 .

The results of the comparative analysis shows that the significant difference in the average value of the lesion before and after treatment with DIAGNOdent Pen for children of 1, 3,6,9 and 12 months of the correspondingages. In a 4-year-olds observed at (2.7 times higher), and the average lesion before treatment was $11,35 \pm 2,41(\mathrm{t}=12,43, \mathrm{p}<0.001)$. At 5 and 6 year olds difference before and after treatment was 2.6 times the average value of the lesions prior to treatment with 5 year is $11,25 \pm 2,47$, and at 6-year was $11,15 \pm 2,23$ (respectively $\mathrm{t}=12,01, \mathrm{p}<0,001$ for 5 years and $\mathrm{t}=12,93, \mathrm{p}<0,001$ for 6 years) Fig. 2.

Discussion: Stationing lesions and their transition from active to inactive tracked for a month. After the first week results of DIAGNOdent pen improved from 15-20 to less than three degrees during this period preventive methods are followed exactly. After the second and third week the results of laser fluorescence decreased to 4 or 3 and any surfaces remained to 5 and 6 of inactive lesions improved by stationing expansion and deepening. Lesion areas with higher levels of laser fluorescence about 11-15, even after successful treatment remained dull Biały, as part of the enamel lesion areas not regained luster, color and the transparency. 
They showed no activity [3] and were stationed with prophylactic and non-invasive treatment for the period of one year.

\section{Conclusions}

1. After the first week results of DIAGNOdent pen improved from the initial value at least three degrees $d 1 b, d 2$ lesions and improved by two grades for d1a lesions.

2. Clinpro TM White Varnish with TCP (Tri-Calcium phosphate) (3M) is effective in reducing demineralization in subsurface layer and in improving the remineralization of surface and subsurface enamel layer.

\section{References}

1. Featherstone JD. Prevention and reversal of dental caries: role of low level fluoride. Community Dent Oral Epidemiol. 1999; 27(1): 31-40.

2. Schmit JL, Staley RN, Wefel JS, Kanellis M, Jakobsen JR, Keenan PJ. Effect of fluoride varnish on demineralization adjacent to brakets bonded with RMGI cement. Am J Orthod Dentofacial Orthop. 2002; 122: 125-130.

3. Yiu CKY, King MN. An overview of dental caries-preventive approaches for children. Hong Kong Dent. J. 2011; 26(8): 29-39.

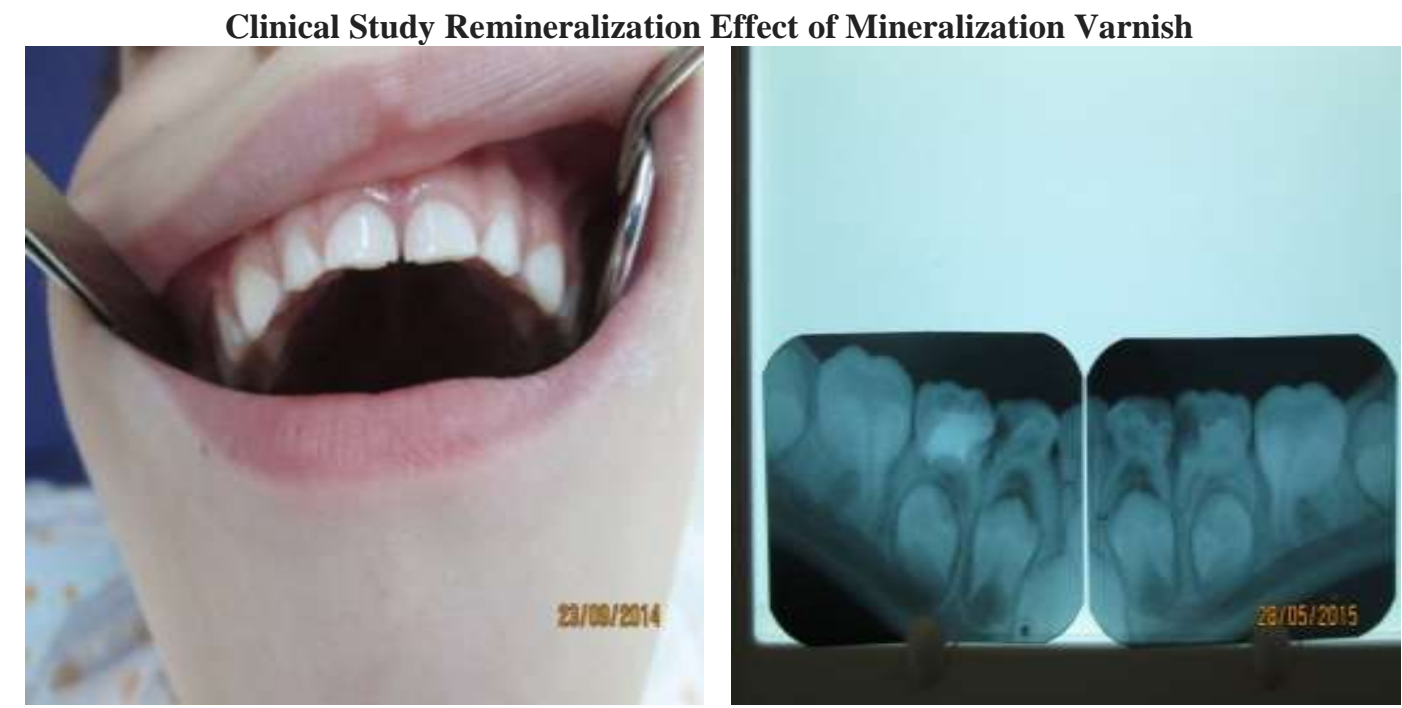

Fig. 1. B. 5 years. Clinical diagnosis and radiographic diagnosis of caries lesions after not invasive treatment on smooth enamel surfaces of the deciduous teeth
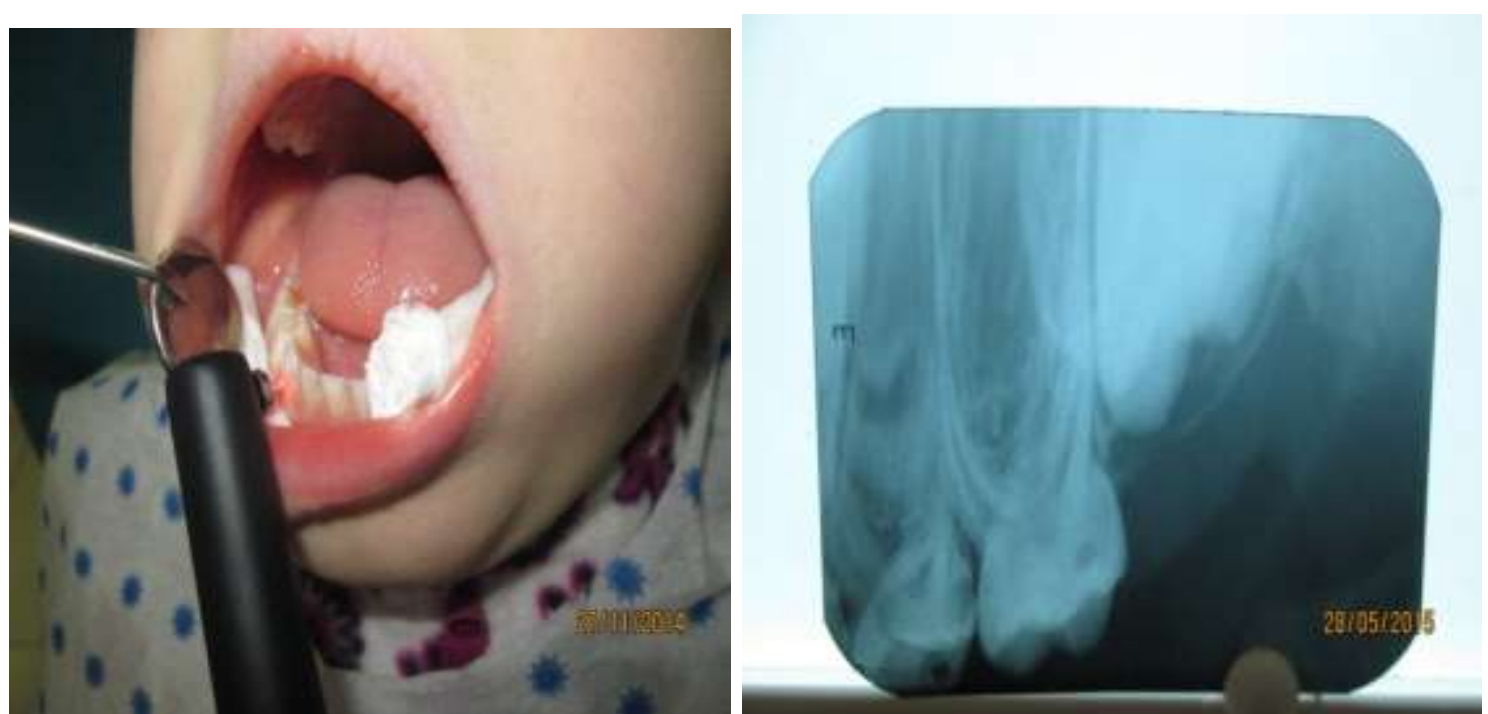

Fig. 2. B. 4 years. Diagnosis of early lesions by DIAGNOdent pen and radiographic diagnosis of d1b lesion in the distal surface of the tooth 54 after not invasive treatment 